\title{
Gradhiva
}

\section{L’idéal du musicien et l'âpreté du monde}

\section{Denis Laborde}

\section{OpenEdition}

Journals

Édition électronique

URL : http://journals.openedition.org/gradhiva/4848

DOI : $10.4000 /$ gradhiva.4848

ISSN : 1760-849X

\section{Éditeur}

Musée du quai Branly Jacques Chirac

\section{Édition imprimée}

Date de publication : 2 septembre 2020

Pagination : $10-23$

ISBN : 978-2-35744-131-6

ISSN : 0764-8928

\section{Référence électronique}

Denis Laborde, "L'idéal du musicien et l'âpreté du monde », Gradhiva [En ligne], 31 | 2020, mis en ligne le 31 mars 2021, consulté le 01 avril 2021. URL : http://journals.openedition.org/gradhiva/4848 ; DOI : https://doi.org/10.4000/gradhiva.4848 


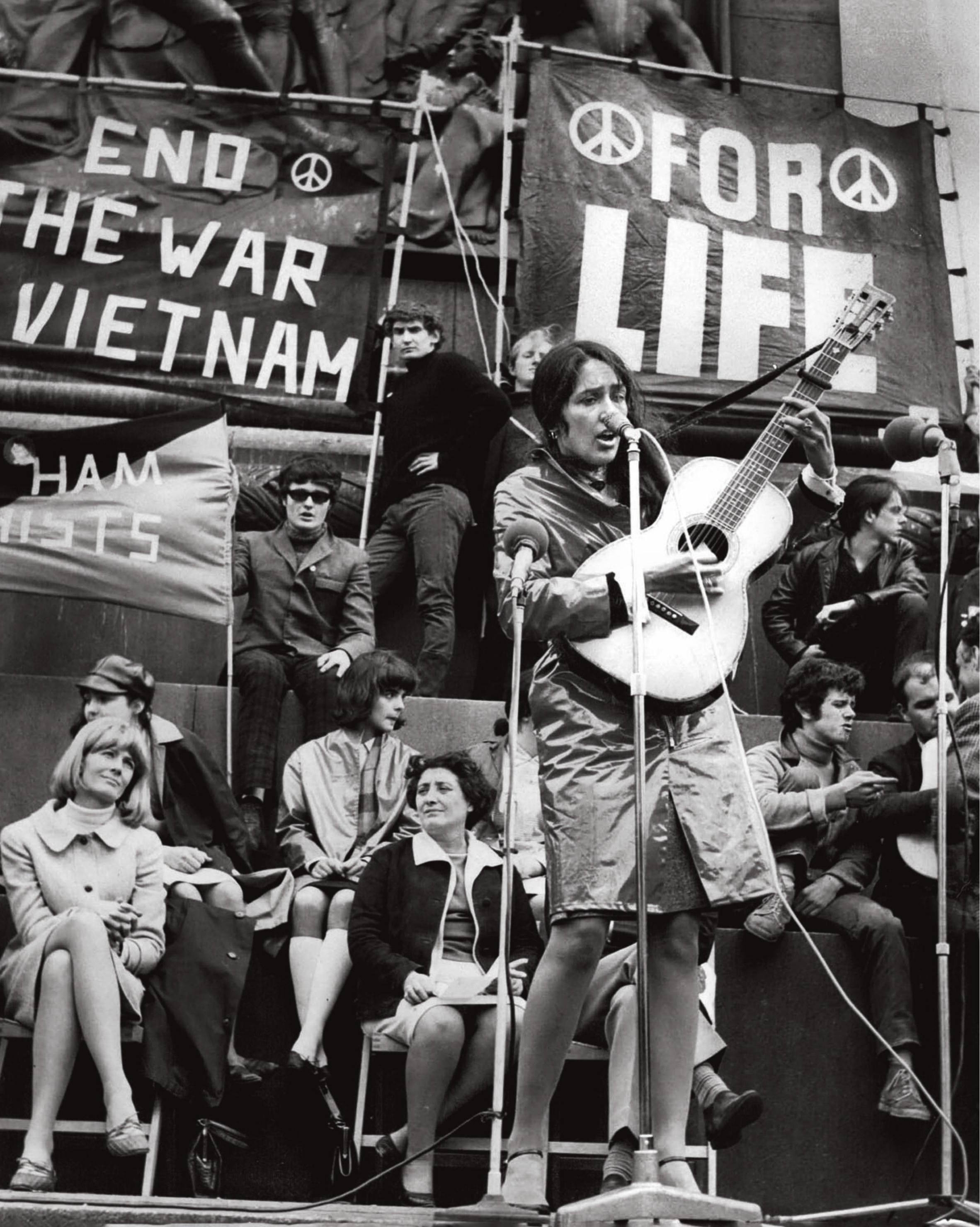




\section{Lidéal du musicien et l'âpreté du monde}

\section{Ci-contre}

Manifestation pour la paix

(détail). ZUMA Press, Inc. /Alamy

Banque d'Images.

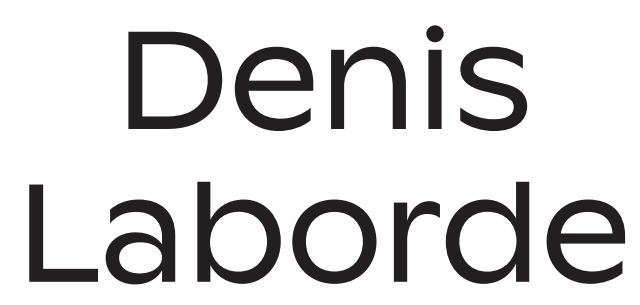

Denis

Laborde 


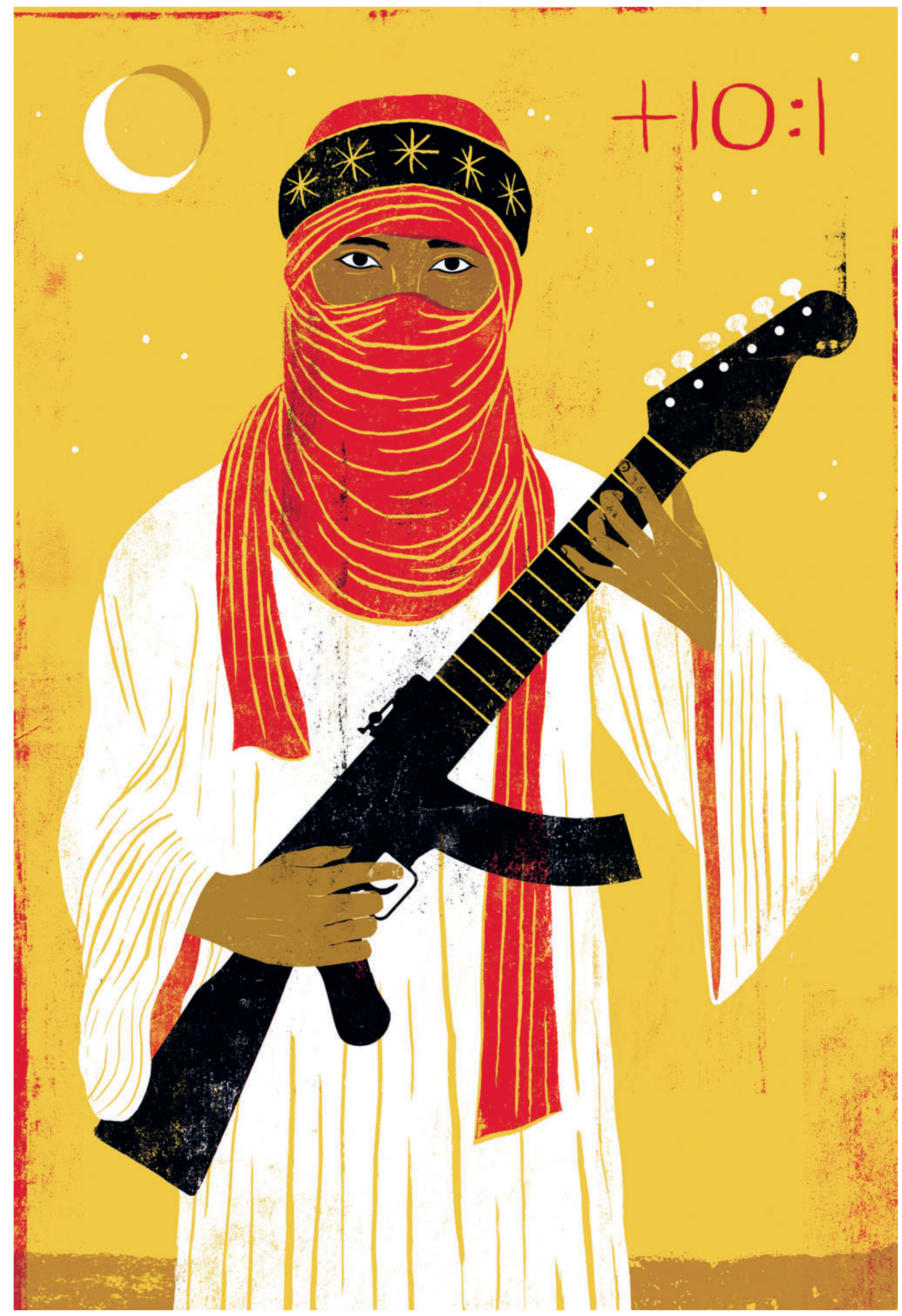

Adrià Fruitós, Tuareg's banishment, 2014. 
Ce numéro de Gradhiva tient son origine du proǵramme des musiques transculturelles de la Fondation Royaumont (95) et des ateliers d'idées qui lui furent associés. La rencontre du même nom orǵanisée le 2 septembre 2017 autour de l'ethnomusicologue Salwa El-Shawan Castelo-Branco fut l'embryon des réflexions déployées ici ${ }^{1}$. Dans cette abbaye cistercienne des confins du Val-d'Oise, haut lieu des sciences humaines et sociales des années 1970, il s'est fabriqué au cours des deux dernières décennies une musique inclassable baptisée «transculturelle» par l'initiateur de ce proǵramme, Frédéric Deval ${ }^{2}$. Son principe était délibérément ingénu: faire se rencontrer à Royaumont des virtuoses porteurs de traditions, leur offrir de travailler ensemble sur un temps long rythmé par des accueils en résidence, puis présenter à Royaumont, mais aussi dans quelques festivals - Banlieues bleues à Saint-Ouen, Aviǵnon, Aix-en-Provence, Villes des musiques du monde à Aubervilliers, Détours de Babel à Grenoble, Haizebeǵi à Bayonne, et encore à Rabat, au Caire ou à New York, ces créations musicales élaborées en commun, «transculturelles» donc ${ }^{3}$.

Cette démarche cultivait la figure d'un musicien en précurseur d'un monde meilleur, partageant une manière de «faire société » ouverte à l'interculturalité, propageant un art bienveillant du vivre-ensemble. Pour cette raison, jamais ce programme ne s'apparenta à la simple gestion d'un secteur d'activité «musiques du monde» au sein des programmes culturels de la fondation. Au-delà des créations partagées, son ambition fut en effet de capter l'attention des institutions partenaires, des mécènes associés et des publics cibles, afin de susciter de leur part une attitude d'écoute, car c'est bien à la condition de s'écouter que l'on pourra s'entendre 4 .

Frédéric Deval présentait communément ces fabrications d'inédits sous l'angle de la rencontre, comme dans cette évocation d'Oración, où la résonance musicienne devient une allégorie de la vie sociale:

\section{Dans le cadre de la création Oración (2014), Amir El Saffar a composé Aswhaaq, une pièce pour quatuor à cordes et voix sur des poésies d'Ibn'Arabi (L'Interprète des désirs). Dans cette composition, le quatuor à cordes, une forme instrumen- tale canonique de la culture musicale européenne, entre magnifiquement en résonance avec les harmonies microto- nales propres aux maqams ${ }^{5}$.}

Nous avons décidé de prendre ici au sérieux l'ingénuité du projet, son ambition irénique, la passion collaborative dont il témoigne, et de mettre en perspective ce programme avec d'autres initiatives qui ont marqué l'histoire des usages sociaux de la musique. Chaque cas examiné confronte l'idéal des musiciens à des configurations sociales, stratégiques, diplomatiques qui disent l'âpreté du monde. Leur mise en série dans le contexte de cette publication ne vise pas à exemplifier une théorie sociale unifiée de la musique, mais à construire une casuistique et à mettre en œuvre les conditions d'un regard partagé sur des usages de la musique en contexte polémologique.

Le monde dont il est question dans ce volume n'est pas le monde géoǵraphique des circulations d'artistes, ni le monde ethnographique des aires culturelles, pas plus qu'il n'est le monde beckerien des sociologies artistiques. C'est le monde continu de l'expérience ordinaire, fait d'épreuves, que Ludwiǵ Wittgenstein définit sur un mode déflationniste comme «alles was der Fall ist ${ }^{\mathbf{6}}$ ", une définition que nous reprenons à notre compte tout en suivant Albert Ogiien dans son invitation à ne pas négliger les interactions qui confèrent aux mots énoncés leurs propriétés compréhensives 7 .

C'est alors dans ce monde des interactions ordinaires que viennent se placer quelques-unes des questions que le programme novateur de Royaumont ne manqua pas de susciter: comment mobiliser la musique pour affronter des situations de violence? Peut-on concevoir que des rencontres musicales agissent sur un monde qui se construit par ailleurs sur un mode belliciste? Les acteurs culturels feraientils semblant de croire à leurs discours comme les Grecs de Paul Veyne croyaient en leurs mythes ou mon fils au Père Noël: pour nous faire plaisir 8 ? Ou pour se forger de bonnes raisons d'agir comme ils aǵissent? Ou pour nourrir l'équivocité du lien supposé entre action altruiste et vertu morale 9 ?

Suffit-il en effet qu'un opérateur culturel invite des combattants américains et irakiens, ennemis sur les terrains d'opération, à élaborer ensemble Sleep Song (2011) à Royaumont pour sceller une réconciliation des pays en guerre? Dans le programme du Festival d'Aviǵnon, Frédéric Deval, ancien de Sciences Po, cherche à nous convaincre :

Sleep Song (2011) a une résonance politique directe avec la guerre d'Irak, avant les bouleversements récents dans les pays arabes. Croisement de poèmes slamés ou performés, construits à partir d'interviezes réalisées par le poète et performeur Mike Ladd auprès de vétérans américains, des poèmes de l'Irakien Ahmed Abdul Hussein et du vétéran américain Maurice Decaul, Sleep Song est porté par un tissu musical composé par Vijay Iyer, pianiste américain d'origine indienne, Serge Teyssot-Gay, guitariste et compositeur français, et Ahmed Mukhtar, joueur d'oud de la diaspora irakienne ${ }^{10}$.

Le programme de Royaumont se tint dans cette ambition que nous questionnons ici. Suffit-il encore de faire se rencontrer les proférations poétiques de Marc Nammour et les musiciens touareg de Tinariwen, qui ont troqué leurs kalachnikovs pour des guitares électriques ${ }^{11}$, pour sceller la rencontre de la parole slam et de toutes les révoltes? De convoquer Kamilya Jubran et sa création Wasl pour réconcilier les Palestine, ou de proǵrammer un tentaculaire Oración
1. Salwa El-Shawan CasteloBranco est professeure d'ethnomusicologie à l'université nouvelle de Lisbonne où elle dirige I'Instituto de

Etnomusicologia, Centro

de Estudos em Música e Dança (Inet-MD). Figure canonique de l'ethnomusicologie, elle préside depuis 2013 I'International Council for Traditional Music (ICTM) voir Estelle Amy de la Bretèque et Victor Stoichiță, «Trois continents, une passion. Entretien avec Salwa El-Shawan Castelo-Branco»», Cahiers d'ethnomusicologie 26, 2013: 241-254). En 2010, elle a coordonné, avec John O'Connell, qui nous accompagne dans ce volume, ce premier ouvrage consacré en propre aux liens entre la musique et les conflits: Music and Conflict, Urbana,

Illinois University Press.

L'atelier d'idées du 2 septembre 2017 à Royaumont fut organisé par Julie Oleksiak et Denis Laborde. II permit de réunir dans la bibliothèque François-Lang de l'abbaye Salwa El-Shawan Castelo-Branco, Talia Bachir Loopuyt Emmanuelle Olivier Loopurt, Marta Amico, John O'Connell,
Frédéric Ramel, Michael Werner (voir «L'idéal du musicien et l'âpreté du monde: construire la diversité par la musique» [en ligne], disponible sur:

https://www.royaumont.com/ $\mathrm{fr} /$ festival17-atelier-d-idees/ presentation). (consulté le 25 juin 2020)

2. Après des études de droit et de sciences politiques, Frédéric Deval (1951-2016) entre dans le groupe Pechiney, mais sa passion pour le cante jondo a raison de son aventure industrielle. Il publie Le Flamenco et ses valeurs (Paris, Aubier, 1989) et rejoint la Fondation Royaumont en 1999. Il y crée le département des musiques orales et improvisées puis le programme des musiques transculturelles (2013), et engage un partenariat avec l'EHESS. Avec Chemirani, Ballaké Sissoko, Djiz, Marc Nammour, Cassol, Magic Malik, Khaled Aljaramani, Kamilya Jubran... il construisit «une famille musicale» (Serge Teyssot-Gay, Le Monde, 4 avril 2016) et fit de Royaumont «le seul lieu au monde où Buenos Aires està vingt mètres de Téhéran» (citation éponyme de l'essai de Gilles Abbeg et Frédéric Deval, Paris, Creaphis 2005). Voir Denis Laborde (éd.), Le Cas Royaumont, Paris, Creaphis, 2014.

3. Parmi les créations de Royaumont: Beyond Black (Nicole Mitchell et Ballaké Sissoko), Oración (Ahmed Essyad, Amir El Saffar, Fawaz Baker), Alefba, Aka Balkan Moon (Fabrizio Cassol), Du Slam à I'Atlas (Andy Emler), Sleep Song (Mike Ladd), À quel dieu parles-tu? (Dgiz, Capitaine Slam/Valère Novarina), Du griot au slameur (Ballaké Sissoko, Lassy King, Médéric Collignon, Andy Emler), The Rhythm Alchemy (Keyvan Chemirani and Family), Le Banquet polyphonique géorgien (Alexandros Markeas/ Ensemble Anchiskhati).

(Voir suite des notes page 14) 
4. Voir Julie Oleksiak, «Des musiques du monde à Royaumont: fabrication de la diversité et programmation de rencontres dans une institution culturelle», thèse de doctorat, cultur EHESS, soutenuele 28 janvier 2020

5. https://www.royaumont. com/fr/amir-elsaffar-tana-stringquartet-khaled-el-hafez/ presentation

(consulté le 25 juin 2020)

6. Que, dans sa traduction française du Tractatus logicophilosophicus, Pierre Klosowski traduit par «tout ce qui arrive» philgentic philosophicus, Paris, Gallimard 1961 [1922]: 29) et que Pierre-

Gaston Granger préfère traduire par «tout ce qui a lieu» Wittgenstein, Tractatus logico-philosophicus, Paris, Gallimard, 1993 [1922]: 33).

7. Les emprunts récurrents au champ sémantique wittgensteinnien furent contemporains de l'avènement d'une sociologie qui endosse le principe de l'ethnographie le principe de l'ethnographi
de terrain afin de saisir les de terrain afin de saisir les prêtée aux capacités de conceptualisation des individus dans la réalisation d'actions en commun participe de ce déplacement de l'espace de ce déplacement de l'espace
logique wittgensteinien vers les épreuves de coordination. Voir Albert Ogien, Les Formes sociales de la pensée: la sociologie après Wittgenstein Paris, Armand Colin, 2007.

8. Paul Veyne, Les Grecs ont-ils cruà leurs mythes? Essai sur I'imagination constituante, Paris, Seuil, 1983.

9. La psychologie sociale a fourni de nombreux modèles d'analys de la motivation altruiste selon que cette motivation vise un objet, un désir ou

une représentation qui guide

I'action. Ici, je me range aux

l'action. Ici, je me range aux
côtés des conclusions de Danie

Batson et de son équipe de

I'université du Kansas lorsqu'il

refuse d'isoler l'altruisme dans un ensemble de motivations qui guident une action. Cette quiguident une action. Ce approche qui lie altruisme et empathie, une approche de sens commun, en somme, qui sépare la question descriptive (comment repère- $t$-on un engagement altruiste?) de question normative (que (quels sont les indicateurs d'un altruisme ultime, ou sincère?) et qui refuse de considérer l'altruisme comme une forme d'égoïsme. Voir Danie Batson, The Altruism Question: Toward a Social-Psychological Anwir New York, Psychology Answer, New York, Psychology Press, 2014.

10. https://www.festivalavignon.com/fr/spectacles /2014/sleep-song (consulté le 25 juin 2020)

11. Voir Marta Amico, La Fabrique d'une musique touarègue: un son du désert dans la World Music, Paris, Karthala, 2020.

(Voir suite des notes page 17)

14
(2015) avec les festivals d'Aviǵnon et d'Aix-en-Provence pour réactiver l'idéal d'une al-Andalus fantasmée en carrefour des trois cultures, érigée par Mahmoud Darwich en métaphore de la Palestine contemporaine: «Et à la fin nous nous demanderons : l'Andalousie futelle Là ou là-bas? Sur la terre... ou dans le poème ${ }^{12}$ ? » Les contributions rassemblées dans ce volume installent cette forme d'altruisme «sur la terre» et examinent des cas qui, à l'instar du programme de Royaumont, eurent l'ambition de faire de la musique un outil pour changer le monde.

\section{UN OUTIL \\ POUR GHANGER LE MONDE?}

Dans la généalogie philosophique de l'Occident, il est d'usage d'ériger Platon en point origine d'une réflexion sur les liens entre musique et politique ${ }^{\mathbf{1 3}}$. Platon puise dans l'étude des proportions musicales formalisées par Pythagore les principes qu'il implémente dans l'organisation politique de la cité: les relations mathématiques à l'œuvre dans l'harmonie musicale sont un pur décalque de l'harmonie des astres. La musique permet ainsi de construire une relation entre le microcosme et le macrocosme, et permet aussi de façonner une âme humaine qui soit en harmonie avec le macrocosme. L'activité musicale devient alors l'outil d'une transposition de cette harmonie dans la vie sociale et politique.

Dans ce numéro de Gradhiva, Michael Werner s'intéresse à la France de l'après-Révolution aux années 1860, en analysant l'usage de la musique dans les projets politiques socialistes naissants et la fonction de démocratisation que le mouvement ouvrier entend lui faire endosser. La mission pacificatrice de la musique est alors au centre des débats qui visent la réorganisation de l'enseignement. À la suite des initiatives pionnières prises en Angleterre par Andrew Bell et Joseph Lancaster, on cherche en Europe les moyens de faire une place à l'enseiǵnement mutuel dans l'organisation générale de l'instruction élémentaire. Michael Werner revient sur la pédagoǵie mutualiste imaǵinée par Guillaume-Louis Bocquillon, dit Wilhem, et son ami Pierre Jean de Béranger, dans le sillage de la Société pour l'instruction élémentaire fondée en 1815. Les idées sur la pratique musicale élaborées dans ce cadre seront en effet reprises par Wilhem, qui publie sa célèbre Méthode élémentaire et analytique de musique et de chant (1821) avant de créer l'Orphéon de Paris (1833). C'est le lancement des sociétés chorales populaires. Cette pratique organisée de la musique entend mobiliser au cœur de la société la fonction pacificatrice de la musique et dépasse largement le cadre français pour se construire dans une perspective croisée, franco-allemande, voire franco-germano-britannique. Saint-simoniens et fouriéristes, Alexandre Choron et Joseph Mainzer portent cette dynamique. Mais l'utopie socialiste s'érode et cet élan vient buter en 1860 sur la nationalisation de la vie musicale européenne, dont la querelle du wagnérisme sera l'une des expressions notoires. S'ouvre alors cette période que Jann Pasler a scrutée dans l'ouvraǵe cardinal qu'elle a consacré à la dimension musicale de l'identité française ${ }^{14}$. À ce moment, nous dit Michael Werner, tout se passe comme si une adhésion patriote au langage musical l'emportait désormais sur les vertus prêtées à la pratique musicienne.

Cette étude de Michael Werner nous fait considérer autrement les pratiques chorales ou orchestrales d'aujourd'hui. Le projet Démos, porté par la Philharmonie, ou l'Orchestre à l'école (trente mille élèves dans mille trois cent trente orchestres) incarnent de nos jours cette confiance prêtée par la puissance publique à la pratique collective de la musique ${ }^{15}$. Démos assume pleinement le rôle qui fut à l'aube du XIX ${ }^{\mathrm{e}}$ siècle celui des sociétés chorales et de l'enseignement mutualiste. La paǵe d'accueil de son site Internet l'explique de façon militante:

Démos (Dispositif d'éducation musicale
et orchestrale à vocation sociale) est un
projet de démocratisation culturelle
s'adressant à des enfants issus de quar-
tiers relevant de la politique de la ville
ou de zones rurales insuffisamment dotées
en institutions culturelles.
Depuis 2010, Démos s'attache à favoriser
l'accès à la musique classique par la
pratique instrumentale en orchestre. Le
dispositif doit sa réussite notamment à
un encadrement éducatif adapté, à la
coopération entre acteurs de la culture
et acteurs du champ social, au dévelop-
pement d'une pédagogie collective spé-
cifique et à la formation continue des
intervenants.
Initié et coordonné par la Cité de la mu-
sique-Philharmonie de Paris, Démos se
déploie aujourd'hui sur le territoire na-
tional grâce à des partenariats avec les
collectivités territoriales ${ }^{16}$.

Et de livrer quelques chiffres : 6000 enfants, et autant d'instruments, 1519 professionnels impliqués dans la structure, 3540 donateurs, 149 concerts en dix ans, 120 heures de pratique collective par enfant, 300 collectivités locales impliquées. Démos suscite l'intérêt jusqu'au sommet de l'État. Le 23 juin 2017, l'Année de la Colombie en France est inaugurée par un concert conjoint des orchestres Démos et Jovenes de Colombia à la Philharmonie de Paris, en présence des présidents Emmanuel Macron et José Manuel Santos. Et c'est depuis la Philharmonie de Paris qu'un an plus tard, le 23 mai 2018, Françoise Nyssen, ministre de la Culture, présente son plan Tous musiciens d'orchestre, qui généralise la pratique musicale et chorale dans les écoles, au principe que "pratiquer un instrument de musique est une ouverture importante sur le monde et sur d'autres méthodes d'apprentissage ${ }^{17}$ ».

À lire les bilans quantifiés, les professions de foi, la structuration administrative du dispositif et l'attention qui lui est prêtée par nos représentants politiques, on pourrait penser que Démos instaure une filiation avec les utopies sociales du XIX ${ }^{\mathrm{e}}$ siècle européen détaillées par Michael Werner. Il n'en est rien. Démos puise 


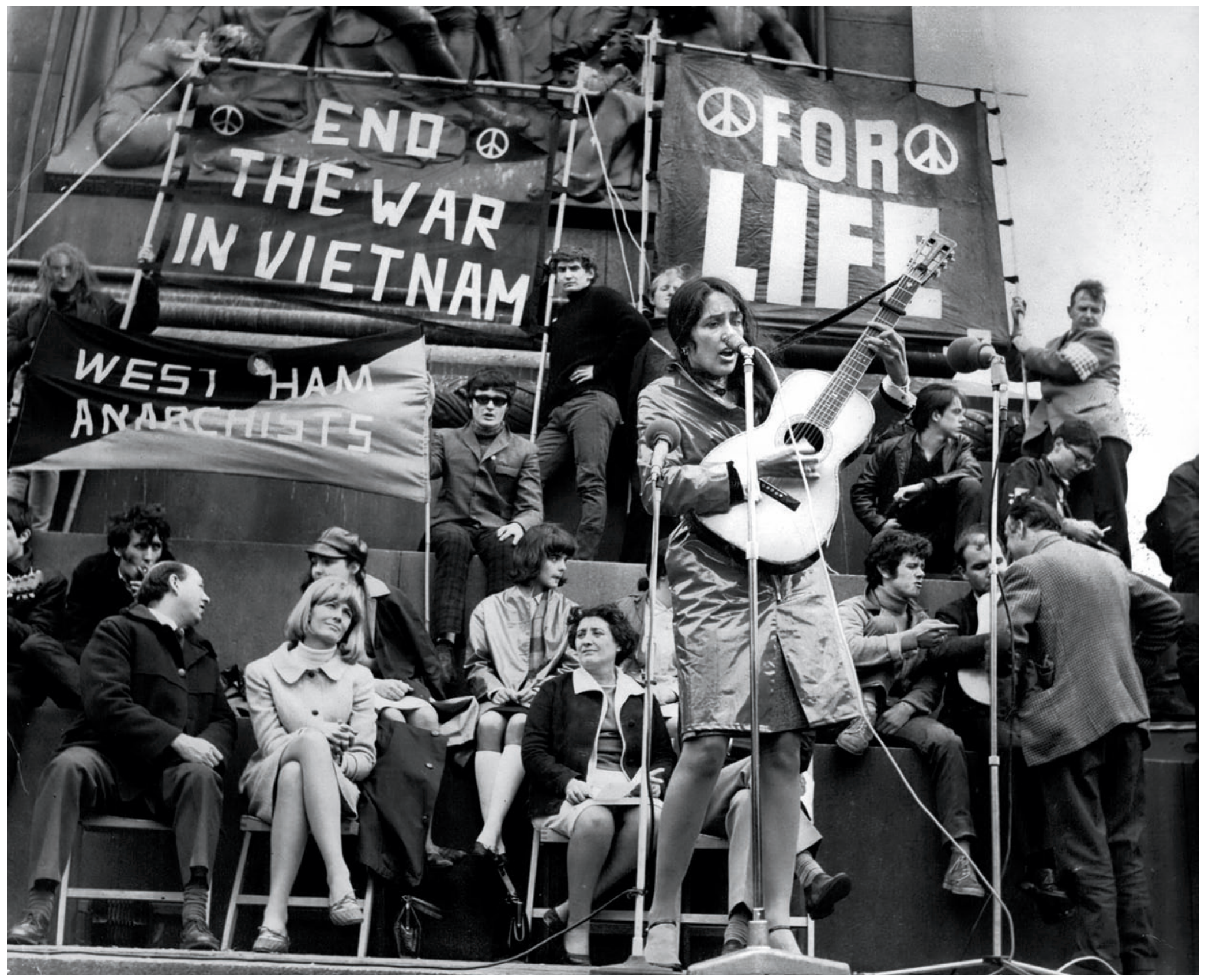




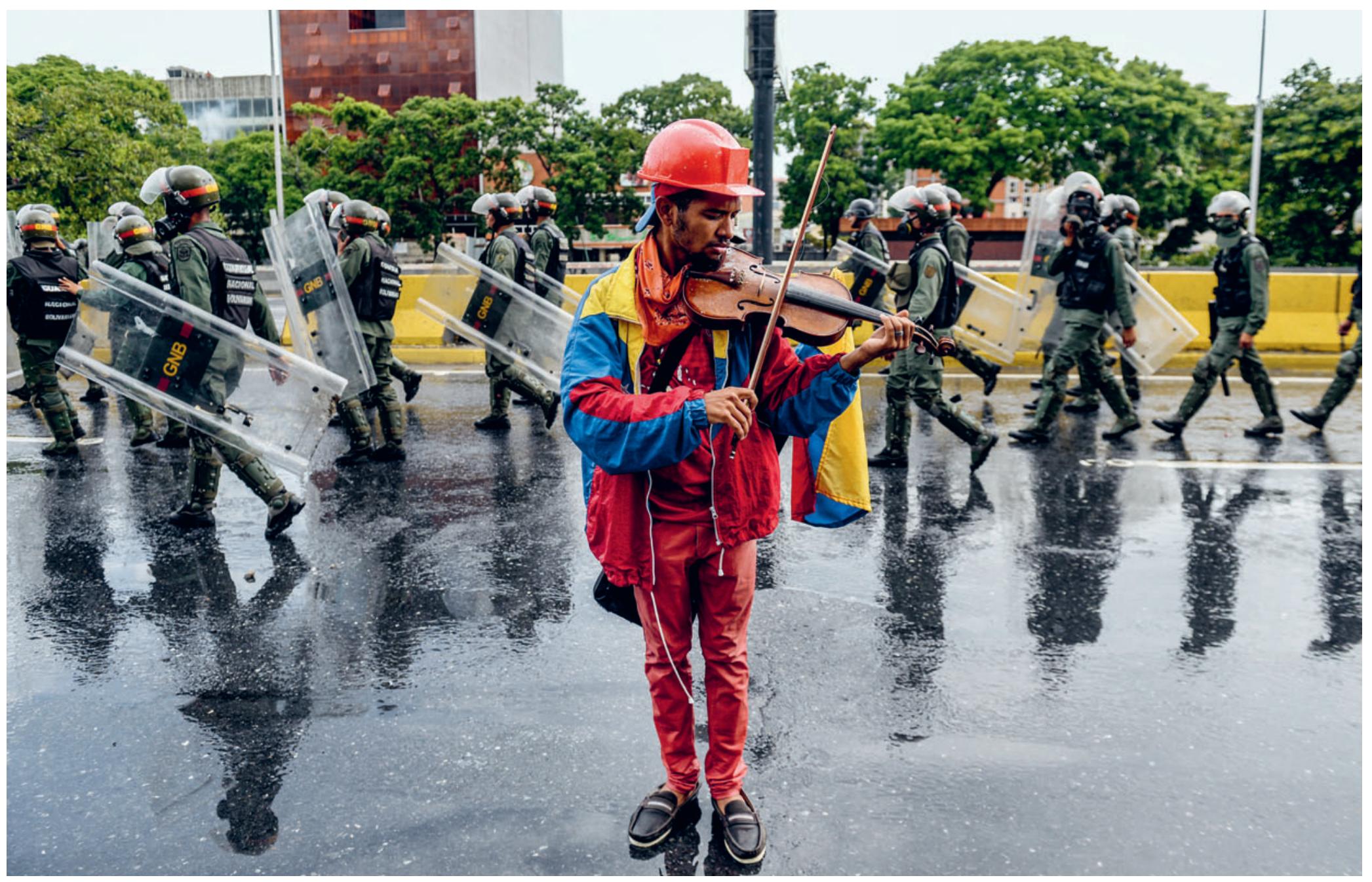

Wuilly Arteaga joue du violon devant la police antiémeute, Caracas, 24 mai 2017. Photo Federico Parra / AFP. 
ses références dans El Sistema, autre projet d'envergure élaboré en 1975 au Venezuela par un économiste chef d'orchestre devenu ministre en 1983, José Antonio Abreu (1939-2018).

El Sistema repose sur un principe qui nous est désormais familier: procurer à des jeunes gens issus de milieux défavorisés un accès à la musique classique par la pratique orchestrale. Le programme a aujourd'hui son icône, le chef d'orchestre Gustavo Dudamel, directeur artistique du Los Angeles Philharmonic. Né en 1981, Gustavo Dudamel est lui-même issu d'El Sistema. La musique fut à ce point importante dans sa vie qu'il considère aujourd'hui qu'elle «doit faire partie des droits humains! La musique, l'art, la culture nous sont aussi nécessaires que la santé, l'éducation... Et pas comme des concepts abstraits, mais pour bâtir sa vie ${ }^{\mathbf{1 8}}$ ». La profession de foi est amplifiée par la littérature journalistique spécialisée et par les ouvrages de musicologie qui considèrent, à de rares exceptions près ${ }^{19}$, qu'El Sistema a valeur de parangon. Mettons en série les titres de quelques ouvrages qui lui sont consacrés: Changing Lives ${ }^{20}$, Playing for Their Lives ${ }^{\mathbf{2 1}}$, Music for Social Change 22, Un Orchestre pour sauver le monde ${ }^{23}$, chacun affichant cet adage du maître Abreu convoqué en quatrième de couverture de la traduction française du livre de Tricia Tunstall: «Si vous mettez un violon dans les mains d'un enfant nécessiteux, il ne prendra pas une arme à feu.»

C'est en répondant très exactement à cette injonction qu'un autre musicien qui a grandi lui aussi dans El Sistema a capté l'attention du monde entier en tentant d'administrer la preuve que la musique est bien un instrument de paix. Durant l'été 2017, alors que le Venezuela se soulève contre le projet d'assemblée constituante du président Maduro, ce violoniste de 23 ans se porte en tête de cortège et s'expose aux assauts de la police antiémeute. Face aux canons à eau et aux bombes lacrymogènes, il joue Bach. Blessé le 22 juillet, arrêté le 27 lors de la grève générale à l'initiative de l'opposition à Caracas, il devient l'icône d'une résistance pacifique avec pour seul objectif de délivrer par la musique «un message de paix ${ }^{24}$ » au cœur même des situations de violence extrême.

Chaque mouvement de protestation produit ainsi ses figures iconiques. À Kiev, de façon constante à partir du 21 novembre 2013, deux cent cinquante mille manifestants se pressent chaque jour sur la place Maidan, rebaptisée par les manifestants Euromaidan ${ }^{25}$. À la suite de la décision prise par le gouvernement ukrainien de renoncer à un accord d'association avec l'Union européenne, la foule des manifestants occupe la place, réclame la démission du président Viktor Ianoukovitch. L'occupation durera trois mois. Des barricades sont construites à l'aide de palissades, voitures, pneus, armoires... et un piano se trouve poussé tout contre.

Élève au conservatoire de Kiev, Antuanetta Mishchenko, alors âgée de 21 ans, refuse que ce piano soit un élément de barricade. Elle décide de le rendre à sa fonction première: produire de la musique. Elle rejoint le mouvement de protestation. Chaque jour, elle monte sur les barricades pour y jouer des études de Chopin.
Les forces de l'ordre ripostent en diffusant des chants patriotiques à fort volume pour couvrir le son du piano. Antuanetta Mishchenko tient bon, d'autres pianistes la rejoignent. On joue Chopin, mais aussi des chants populaires, Let It Be... tout ce que chacun a dans les doiǵts. «Je fais ce que l'on attend de moi, dira-t-elle plus tard. C'est important pour moi de me sentir utile. Je leur ai done donné tout ce que je pouvais ${ }^{\mathbf{2 6}}$. Bien sûr, il y eut un prix à payer. L'exercice quotidien dans l'hiver kiéviste eut raison de ses doiǵts gelés. De longs mois furent nécessaires pour que ses mains redeviennent des mains de pianiste. Mais à mesure des jours et des nuits de veille et d'affrontements, «le piano de Kiev» peint aux couleurs du pays est «devenu symbole de la révolution, de la résistance pacifique», déclare Markiyan Matsekh, qui coordonna cette mise en relation de l'idéal des pianistes avec la rudesse de ces affrontements armés qui firent cent vinǵt morts ${ }^{\mathbf{2 7}}$.

\section{MUSICIENS DES CAMPS}

Le lien ente pratique musicale et pacification sociale peut aussi se construire sur un mode d'émancipation. C'est à la cause palestinienne que Nicolas Puiǵ consacre sa contribution à ce volume. Cette cause est soutenue par des mouvements politiques, des mouvements sociaux, elle est installée dans des rapports de force militaires, diplomatiques, géostratégiques, elle est relayée aussi par des formes d'engagement artistique. Dans le camp de Burj el-Barajneh, banlieue sud de Beyrouth, Nicolas Puig a rencontré les rappeurs palestiniens du groupe Katibeh Khamseh. Pour eux, l'engagement politique n'est pas tout. Ils sont habités par «la cause du rap». Ils se tiennent ainsi dans cette tension entre le souci politique d'œuvrer à la construction d'un État palestinien et l'impératif artistique de cultiver la force émancipatrice du rap, alors même que, pour leur communauté d'appartenance, cette pratique est entachée de dépravation. "It's not an easy country», nous confie Amru (nom de scène: Osloob). Ils décident de fuir.

Nicolas Puig, leur propose de filmer l'exil, de Beyrouth à Londres. Il confie à l'un d'eux une caméra de poing: qu'il filme donc ce qui pourra l'être. Le résultat est saisissant. Le dispositif nous confronte à la question de la circulation des artistes, à l'asymétrie Nord-Sud, et sert de révélateur des procédures d'accueil et des multiples étapes qu'il leur aura fallu franchir pour être considérés, statutairement, comme des rappeurs palestiniens experts en leur art, et non plus comme des miǵrants musiciens sur les routes de l'exil.

Les formes artistiques, les genres musicaux, les choix esthétiques constituent-ils des domaines réservés? Comment les opérations d'évaluation, de jugement, d'assignation statutaire se déroulent-elles?

La contribution de John O'Connell s'intéresse à la disponibilité non pas des statuts mais des formes culturelles elles-mêmes.
12. La terre nous est étroite et autres poèmes: 1966-1999, trad. de l'arabe (Palestine) par Elias Sanbar, Paris, Gallimard, 2000 [Chant 1].

13. «Platon, le premier, exprime l'exigence de maîtriser la musique et de la mettre au service du courage et de la vertu en en excluant les effets nostalgiques et amollissants (Platon, La République, 424 b-c; Les Lois, 659e-660a, 812a-d). Ces prescriptions sont en rapport avec les conceptions métaphysiques et éthiques du beau développées dans le Timée et dans le Philèbe: la relation entre la beauté cosmologique et celle de l'âme s'instaure à partir des principes fondateurs du monde reposant sur la symétrie, la beauté étant, avec la proportion et la vérité, l'une des trois

caractéristiques du bien (Platon,

Philèbe, 64d-65a; Timée, 87c et 90 a) et, en raison des effets objectifs des différents modes musicaux, il convient d'en maîtriser l'usage aux fins de l'unité politique et de la formation du citoyen (Jean-Marie Donegani, citoyen (Jean-Marie Donegani, «Musique et politique: le langage musical entre expressivité et vérité», Raisons politiques 14 , 2004: 5-19).

14. Jann Pasler, La République, la musique et le citoyen. 1871-1914, trad de l'anglais par JohanFrédérik Hel Guedj, Paris Gallimard, 2015 [2009].

15. Gilles Delbarre et Denis Laborde (dir.), Démos: genèse acteurs, enjeux, Paris, Éditions de la Philharmonie, 2019.
.

16. https://demos.

philharmoniedeparis.fr/le-projet. aspx (consulté le 28 mai 2020).

17. https://www.culture.gouv. $\mathrm{fr} /$ Presse/Dossiers-de-presse/ Plan-Tous-musiciens-d-orchestre (consulté le 4 février 2020).

18. Sophie Bourdais, «Gustavo Dudamel, chef d'orchestre:

"Tout ce que je possède dans ma vie est relié à la musique" », Télérama, 18 mars 2016 [en ligne], disponible sur: https://www. telerama.fr/musique/gustavodudamel-chef- $d$-orchestretout-ce-que-je-possede-danstout-ce-que-je-possede-dans-
ma-vie-est-relie-a-la-musique, 139827.php

(consulté le 17 juin 2020).

19. Je fais ici mention de l'ouvrage critique du journaliste Geoffrey Baker, qui s'étonne de ce qu'El Sistema fasse de l'orchestre symphonique un modèle d'émancipation individuelle, alors même qu'il repose sur un modèle d'éducation coercitive dans un continent marqué pourtant par des propositions pédagogiques réellement émancipatrices, comme celles du Brésilien Paulo Freire (1921-1997) et sa Pédagogie des opprimés, qui permettent de mettre en œuvre des dispositifs mettre en œuvre des dispositifs
d'enseignement mutualisé se situant bien loin de la stricte obéissance au chef. Voir Geoffrey Baker, El Sistema: Orchestrating Venezuela's Youth, Oxford, Oxford University Press, 2014.

(Voir suite des notes page 20) 


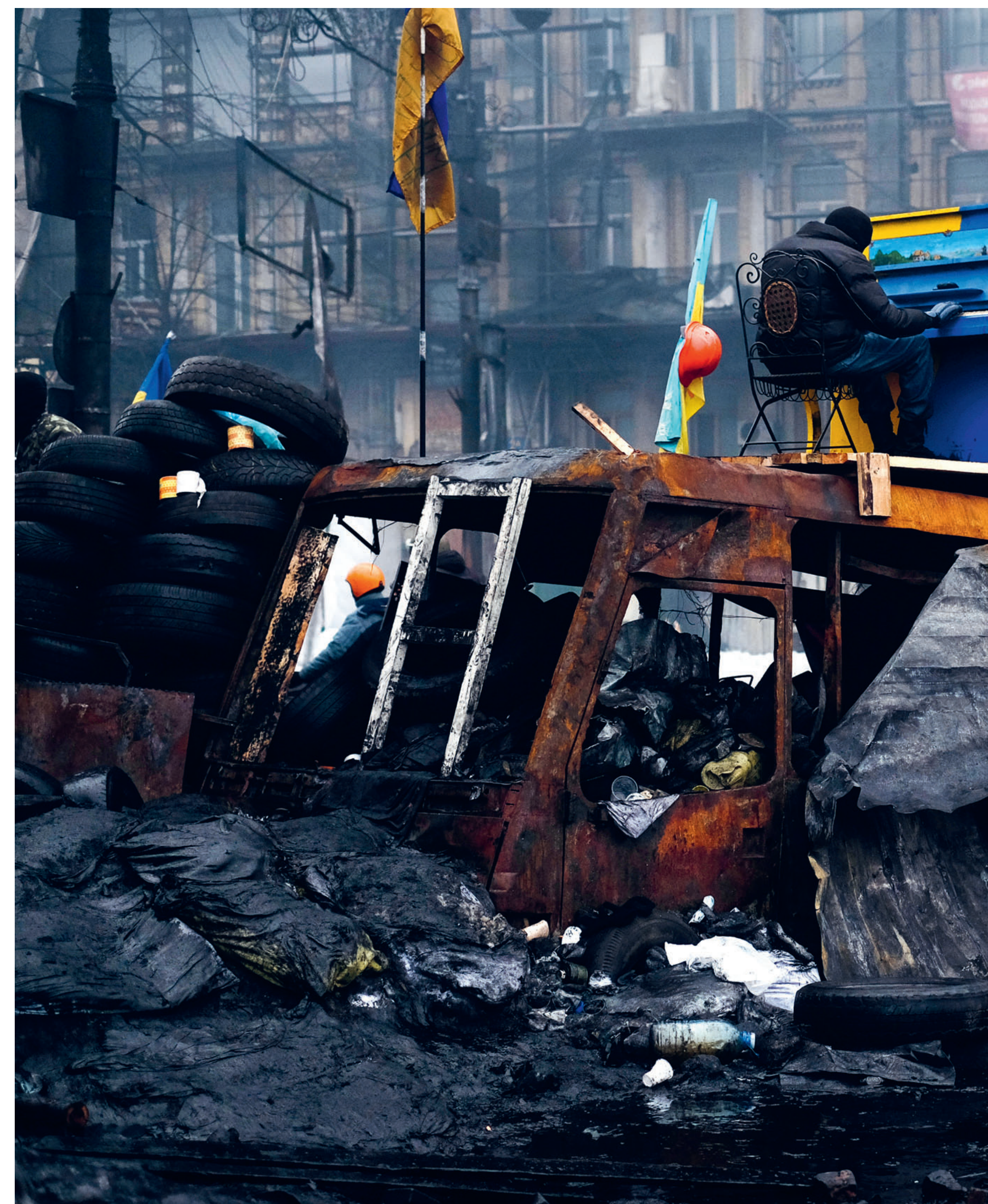







20. Tricia Tunstall, Changing Lives: Gustavo Dudamel, El Sistema, and the Transformative Power of Music, New York, Norton and Company, 2012 (trad. en français par Cécile Roure sous le titre Changer des vies par la pratique de l'orchestre: Gustavo Dudamel et l'histoire d'El Sistema, Lyon, Symétrie, 2015).

21. Tricia Tunstall et Eric Booth Playing for Their Livres: Th Global El Sistema Movementfor Social Change Through Music, New York, Norton and

Company, 2016.

22. Christine Witkowskl (dir.) El Sistema: Music for Social Change, New York, Overlook Omnibus, 2016.

23. Vincent Agrech, Un orchestre pour sauver le monde: voyage au cœur du Sistema. Du

Venezuela à la France, le miracle de l'éducation par la musique,

Paris, Stock, 2018.

24. «Au Venezuela, le violoniste Wuilly Arteaga a été libéré», Le Monde, 16 août 2017 libéré», Le Monde, 16 aou
[en ligne], disponible sur: https://www.lemonde.fr/ ameriques/article/2017/08/16/ au-venezuela- le-violonistewuilly-arteaga-a-ete-libere $51727693222 \mathrm{html}$

(constulté le 3 septembre 2019).

25. Benoît Vitkine, «Des centaines de milliers d'Ukrainien réclamentle départdu président Le Monde 8 décembre 2013 sponible sur: https://www.lemonde.fr/ europe/article/2013/12/08/ en-ukraine-l-oppositionappelle-a-une-mobilisation massive 3527504 3214 htm (consultéle 12 mars 2020).

26. Anastasia Boichenk «Antoinette Mischenko, The Pianist: "Playing classical music is reflecting your own vision of the world through other's ar pieces"», Opinion, 26 octobre 2018 [en ligne], disponible sur: 2018 [en ligne], disponible sur:
https://opinionua.com en / 2018 https://opinionua.com en /2018 playing-classical-music/ consulté le 17 juin 2020).

27. Voir le film documentaire de la réalisatrice Vita Maria Drygas: Piano (Pologne, 2015, 45 min.), prix des Jeunes aux Escales documentaires de La Rochelle.

28. John Blacking, Le Sens musical, trad. de l'anglais par Éric et Marika Blondel, Paris, Edition de Minuit, 1980 [1973]: 101

29. Jacques Cheyronnaud «Sacré à plaisanterie. Référence religieuse et disponibilité culturelle», Protée 27, 1999:77-92

30. Esteban Buch, La Neuvième de Beethoven: une histoire politique, Paris, Gallimard, 1999: 308.

\section{DISPONIBILITÉ \\ DES FORMES GULTURELLES}

Si l'on s'accorde à considérer avec l'ethnomusicologue John Blacking que puisque la musique est "du son organisé, elle exprime des aspects de l'expérience des individus en société $\mathbf{2 8}$, force est de constater que «ce que dit la musique» est moins à chercher dans l'énoncé musical que dans la forme relationnelle construite dans l'énonciation, de façon contextuelle et historicisée, ce qui fait d'elle une forme culturelle. L'histoire de la musique est parsemée de thèmes, de mélodies, de citations qui portent témoignage des mésusages, des mécompréhensions et des polémiques liées à ce que Jacques Cheyronnaud nomme «la mise en disponibilité culturelle 29 » de la musique pour indiquer toutes les formes distinctes d'appropriation auxquelles elle peut donner lieu. Les travaux d'Esteban Buch sur la Neuvième Symphonie de Beethoven ont montré que l'Ode à la joie, par exemple, tenue comme «le premier emblème musical de la valeur morale de l'art », a fait l'objet d'appropriation guerrière de la part des nazis, qui sont «la principale incarnation historique du $\mathrm{Mal}^{\mathbf{3 0}}$ ». L'histoire de la musique est également jonchée de décrets, de bulles papales, de procès, de fatwas qui mettent en cause des usages de la musique jugés déviants, et s'efforcent de maintenir le bon usage ${ }^{31}$ C'est à l'un de ces cas que l'ethnomusicologue britannique John O'Connell prête attention dans ce volume.

Spécialiste de l'Empire ottoman, John O'Connell avait publié avec Salwa El-Shawan Castelo-Branco l'ouvrage fondateur Music and Conflict (voir supra, note 1). Sous un titre malicieux, E Pluribus Unum ${ }^{32}$, il s'intéresse à la façon dont une mélodie peut être livrée à des formes divergentes d'appropriation en régiime hymnique martial au début de la Première Guerre mondiale. Deux marches militaires, l'une arménienne, l'autre turque sont chantées sur une même mélodie ${ }^{33}$. La première fédère un consensus hétérogène et impérialiste, la seconde vient promouvoir un nationalisme dissident. Ainsi une même mélodie sert-elle des desseins antagoniques : anticiper le pluralisme d'une société inclusive d'une part, promouvoir une forme de patriotisme exacerbé dans ce moment critique de l'histoire de la Turquie d'autre part.

Ce lien entre musique, hymne et nationalisme est également travaillé dans un tout autre contexte par Jaime Salazar, qui se concentre sur le Plan Nacional de Música para la Convivencia qui fut élaboré par le gouvernement colombien en 2002. Cette réforme politique s'inscrit dans une série de plans élaborés par les gouvernements successifs destinés à valoriser par la musique la grande diversité culturelle du pays et faire de ses traditions musicales l'instrument d'une paix sociale dans un contexte de violence endémique. Jaime Salazar explique que nous n'échappons pas à la construction de formes figées stéréotypées (le bullerengue de Petrona Martínez, par exemple, dans la régiion de Bolívar, sur la côte caraïbe), mais la confiance dans la possibilité d'user de la musique comme d'un instrument de paix favorise aussi l'émergence de formes inventives d'organisation sociale à travers des initiatives locales singulières (la Fondation Tumac de la famille
Tenorio à Tumaco, «la perle du Pacifique», dans la région du Nariño à la frontière équatorienne, qui, avec ses trente mille hectares, compte le plus grand nombre de plantations illégales de coca). Pour autant, Jaime Salazar ne se contente pas d'étudier les textes de loi ou d'énumérer la prolifération des initiatives locales. Il mène l'enquête au sein du ministère de la Culture. En rencontrant les fonctionnaires, en examinant avec eux la rationalité des formes d'organisation administrative, il scrute la façon dont la musique s'inscrit au cœur des politiques culturelles, dont les réseaux régionaux se structurent en lien avec les services de l'État et dont les fonctionnaires instruisent une continuité dans la mise en œuvre de ces politiques. L'effet induit est paradoxal: il garantit l'efficacité de l'action administrative qui maintient un dialogue avec les acteurs locaux par-delà les changements imposés par l'alternance politique.

\section{LA MUSIQUE POUR CHANGER LE MONDE}

Si la musique peut être l'instrument d'une action politique au sein d'un appareil d'État, elle peut aussi devenir un outil stratégique dans le cadre de relations diplomatiques. La présence du violoncelliste Mstislav Rostropovitch (1927-2007) jouant les suites de Bach à Check Point Charlie a produit l'une des images iconiques de cette séquence majeure de l'histoire du $\mathrm{Xx}^{\mathrm{e}}$ siècle que fut la chute du mur de Berlin. Le 9 novembre 1989, les autorités est-allemandes décident de ne plus soumettre à autorisation préalable le passaǵe à l'Ouest. Aussitôt, les Berlinois de l'Est entament la destruction du mur. Les images font le tour du monde. Rostropovitch est à Paris, il file à Berlin. Le 11 novembre, le voici incognito devant le mur, jouant les suites de Bach. Chacun des présents sort son appareil photo. Quelques années plus tard, en 1997, il commente l'une de ces photos pour la journaliste du Monde Annick Cojean:

\section{Toute ma vie est là-dedans. Ma cohérence, mon unité. Mais qui pourrait com- prendre? C'est mon histoire à moi. Et ce que je célébrais, ce jour de novembre 1989, c'était la réunification des deux parties de ma vie dont le Mur odieux symbolisait la déchirure. D'un côté de la Muraille se trouvaient mon passé, mon pays, mes racines; de l'autre côté mon exil, mon travail, mon avenir. Deux pans de vie cloisonnés, hermétiques, que j'avais cru ne jamais pouvoir réunir et qui me don- naient le sentiment d'être amputé, incomplet $^{\mathbf{3 4}}$.}

Rostropovitch est l'icône des enjeux diplomatiques de la guerre froide. Fragilisé en raison de son amitié avec le romancier Alexandre Soljenitsyne, prenant la défense d'Andreï Sakharov et de nombreux opposants au régime soviétique, il fut marginalisé dans son propre pays avant de devenir un enjeu des relations 
diplomatiques. Au mois de janvier 1974, l'Unesco s'apprête à célébrer le $25^{\mathrm{e}}$ anniversaire de l'International Music Council, son orǵane consultatif sur les questions musicales. À ce moment, l'IMC est présidé par une autre figure charismatique de la musique, le violoniste Yehudi Menuhin (1916-1999). Menuhin décide d'inviter Rostropovitch à Paris. Leonid Brejnev s'y oppose. Un rapport de force s'installe. L'URSS proteste auprès de l'Unesco, puis cède: un visa est accordé au violoncelliste qui joue à Paris le 8 janvier. Quelques mois plus tard, Rostropovitch s'exile à l'Ouest; en 1978, il est déchu de sa citoyenneté soviétique.

C'est au cœur de ces tensions diplomatiques que nous plonge l'historienne Anaïs Fléchet. Elle déchiffre pour la première fois ici les archives privées de Yehudi Menuhin, recoupe ces informations avec celles recueillies dans les archives de l'International Music Council, confronte ces données aux sources diplomatiques américaines et aux Archives russes d'État de la littérature et des arts. À la croisée de ces sources multiples, elle dresse un portrait du musicien en diplomate. Faisant de l'art "un espoir pour l'humanité 35 », il a installé au cœur de la guerre froide une diplomatie non gouvernementale qui prend la musique pour instrument, et a trouvé comme héritiers Bono, Daniel Barenboïm ou Yo-Yo Ma, dont Frédéric Ramel brosse le portrait dans l'entretien qu'il nous accorde en conclusion de ce dossier thématique.

Frédéric Ramel dirige à Sciences Po le département de science politique. L'ouvrage qu'il a coordonné avec Cécile Prévost-Thomas en 2018 (International Relations, Music and Diplomacy ${ }^{36}$ ) fait de lui l'un des pionniers de l'étude des usages contemporains de la musique dans les relations internationales ${ }^{37}$. Au cours de notre entretien, il caractérise trois formes d'engagement musicien dans l'action diplomatique: une diplomatie des célébrités (Bono, leader charismatique du groupe irlandais U2, qui met ses concerts et sa célébrité au service de l'ONG ONE pour lutter en Afrique contre l'extrême pauvreté et les maladies évitables ${ }^{38}$ ), une diplomatie à voies multiples, incarnée par Daniel Barenboïm et le théoricien littéraire Edward Saïd, qui prennent leur part dans le dialogue israélo-palestinien en créant à Weimar en 1999, le West-Eastern Divan Orchestra ${ }^{39}$, permettant à quatrevingts musiciens de Palestine, d'Israël et des pays voisins de venir en Europe chaque été pour une session de répétitions en formation symphonique et une tournée de concerts dans les villes du pourtour méditerranéen ${ }^{40}$. Enfin, la diplomatie transprofessionnelle incarnée par Yo-Yo Ma et son Silk Road Project ${ }^{41}$ contraste avec la précédente. Contrairement à Bono ou à Barenboïm qui situent leurs actions au cour d'enjeux politiques internationaux identifiés, Yo-Yo Ma entreprend une action culturelle transnationale, qui repose sur la mobilisation de musiciens locaux et sur la programmation de formes classiques de la circulation musicale (concerts, formations, animations scolaires).

Cette caractérisation de l'action diplomatique qui prend au sérieux la force transformatrice de la musique a sans doute ses limites. C'est qu'il en va ici de la musique comme il en est ailleurs de la vérité.

\section{TERREURS}

SONORES

«Le vrai ne dévoile pas l'éniǵme du tenir pour vrai ${ }^{42}$ », nous rappelle Gérard Lenclud. De même, la force pacificatrice d'une musique ne dévoile pas par elle-même l'éniǵme du tenir une musique pour pacificatrice. Ce qui vaut pour la vérité semble donc bien valoir pour la musique: considérer une musique sous l'anǵle de sa fonction pacificatrice ne dit rien de l'engagement dans la croyance qu'une musique est pacificatrice.

Cette éniǵme empêche d'adhérer à l'idée que la musique aurait pour seule fonction de servir de nobles desseins de concorde. Il arrive en effet que la musique se trouve mobilisée pour de terribles projets. Les hymnes constituent en cela des entités remarquables qui invitent au combat avec la mort pour horizon désirable, mais on trouve de nombreux cas qui mettent la musique au service de l'intolérance, du racisme, de l'exclusion : «Kill all battyman», clame-t-on dans les dancehalls de la banlieue de Kingston à l'unisson avec les hérauts de l'homophobie que sont Beenie Man, Bounty Killer ou Capleton et son tube More Prophet ${ }^{43}$ Et dans le chapitre qu'il consacre ici à la Colombie, Jaime Salazar explique qu'au moment où les gouvernements successifs s'efforcent de faire de la musique l'instrument d'une paix sociale encore largement à construire, les paramilitaires des Autodefensas Campesinas de Córdoba y Urabá (Accu) jouent de la musique pour accompaǵner la plus terrible des atrocités. C'est le massacre du Salado. Entre le 16 et le 21 février 2000, dans le villaǵe d'El Salado, dans les Montes de María (Bolívar), quatre cent cinquante hommes arborant le brassard des Accu exécutent en musique soixante-six victimes.

De tels usages de la musique sont aujourd'hui bien documentés. De nombreuses études portent sur les espaces concentrationnaires ${ }^{\mathbf{4 4}}$, et Suzanne Cusick a élaboré les premières recherches portant en propre sur l'usaǵe de la musique dans des pratiques de torture 45 En 2017, dans l'ouvrage qu'il consacre à Helmut Lachenmann, Laurent Feneyrou s'intéresse à l'usage de la musique par les services de la police allemande pour torturer Gudrun Ensslin, membre fondatrice de la Fraction Armée rouge (RAF), et amie du compositeur ${ }^{46}$. Ses travaux prennent appui sur l'ouvrage magistral que Steve Goodman a consacré à la guuerre sonique en mettant en série des situations dans lesquelles des sons sont destinés à produire une «écologie de la peur ${ }^{47}$ ». Il est relayé par J. Martin Daughtry qui a produit une étude de référence sur la guerre d'Irak ${ }^{48}$. Il est vrai que, des trompettes de Jéricho aux procédures de torture acoustique à Guantanamo, le pouvoir de la musique fait l'objet d'un intérêt toujours renouvelé par les acteurs de la guerre. La journaliste Juliette Volcler a composé un essai d'importance sur les usages militaires de la musique comme alarme directionnelle servant les technologies «non létales» de contrôle des foules ${ }^{49}$. Et Luis VelascoPufleau, après avoir produit l'étude de référence sur les attentats du Bataclan ${ }^{\mathbf{5 0}}$, vient de coordonner la publication d'un remarquable numéro de la revue
31. Voir par exemple: Denis Laborde, «The Unbearable Sound: The Strange Career of Musicoclashes», in Bruno Latour et Peter Weibel (dir.), Iconoclash: Beyond the Images Wars in Science, Religion and Art, Cambridge, MIT Press, 2002: 253-280.

32. Que I'on peut traduire par «faire un à partir de plusieurs», ce qui est aussi la devise gravée sur les pièces de monnaie américaines depuis 1795 .

33. On parlerait ici de timbre musical. Le folkloriste Patrice Coirault a en effet proposé de donner à une mélodie qui se prête à de multiples emprunts le nom de «timbre», et d'appeler ainsi «tout air, vocal ou instrumental, préexistant aux paroles qui s'y joignent pour faire morceau de chant ou former une chanson $\gg$ (Patrice Coirault, Notre chanson folklorique, étude d'information générale: l'objet et la méthode, l'inculte etson apport, l'élaboration, la notion, Paris, Auguste Picard, 1941: 207, n. 2).

34. Annick Cojean, «Berlin, 1989: le maestro et le Mur», Le Monde, 8 novembre 2019 [en ligne], disponible sur: https:// www.lemonde.fr/international/ article/2019/11/08/berlin-1989le-maestro-et-le-

mur_6018497_3210.html (consulté le 20 mars 2020).

35. Je me permets cette allusion à son recueil de textes: Yehudi Menuhin, L'Art, espoir pour I'humanité: discours et écrits, trad. de l'allemand par Sylvie Escat, Paris, Buchet-Chastel, 1999 [1987].

36. Frédéric Ramel et Cécile Prévost-Thomas (dir.) International Relations, Music and Diplomacy: Sounds and Voices on the International Stage, Cham (Suisse), Palgrave Macmillan, 2018.

37. Parmi les développements récents de ces travaux: Rebekah Ahrendt, Mark Ferraguto et Damien Mahiet (dir.), Music and Diplomacy from the Early Modern Era to the Present, New York, Palgrave Macmillan, 2014; Jessica Gienow-Hecht (dir.), Music and International History in the Twentienth Century, New York/ Oxford, Berghahn Books, 2015.

38. https://www.one.org/fr/ (consulté le 25 juin 2020)

39. https://west-eastern-divan org/ (consulté le 25 juin 2020)

40. L'histoire de cette rencontre entre Daniel Barenboïm et Edward Saïd est détaillée dans le recueil d'articles écrit à deux voix publié en traduction française en 2003: Edward Saïd et Daniel Barenboïm, Parallèles et paradoxes: explorations musicales et explorations musicales et
politiques, Ara Guzelimian (éd. et préf.), trad. de l'anglais par Philippe Babo, Paris, Le Serpent à Plumes.

(Voir suite des notes page 23) 


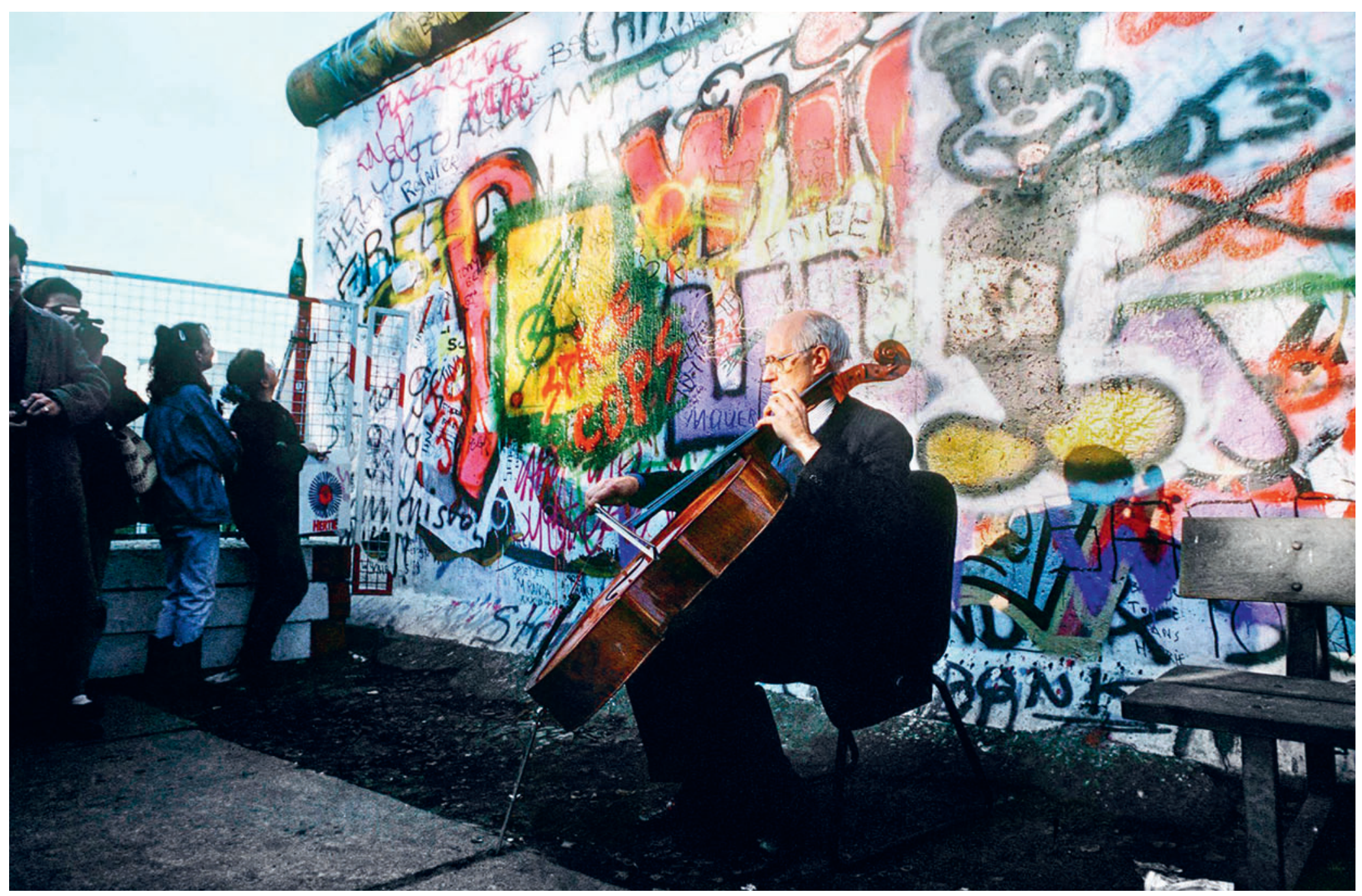

Mstislav Rostropovitch jouant un morceau de Johann Sebastian Bach devant le mur de Berlin, non loin de Checkpoint Charlie, 9 novembre 1989. SUCCO / ACTION PRESS / VISUAL Press Agency. 
Transpositions sur le triptyque: son, musique et violence ${ }^{51}$. On ne trouvera pas dans les pages suivantes de travaux relevant de ces usages de la musique. De même qu'il n'existe que «des programmes hétérogènes de

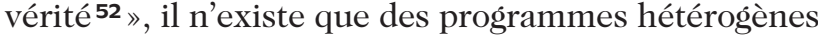
de mobilisation musicale. Les analyses convoquées ici ne concernent que des programmes qui cultivent l'idée d'une musique érigée en outil de transformation des sociétés humaines.

Ce numéro de Gradhiva est aujourd'hui publié dans le contexte troublé d'une pandémie qui interroge profondément nos façons de vivre en société. Je rédige ces liǵnes au sortir d'une longue période de confinement pendant laquelle nous fîmes silence, les opéras, les théâtres, les cinémas furent fermés, et nos espaces publics rendus silencieux par la contrainte des déplacements dérogatoires. Pourtant, "de la musique» est venue s'immiscer dans les interstices de nos confinements connectés comme en lisière de nos aires de clôture. Une sociabilité musicale des balcons s'est déployée dans nos rues qui résonnaient chaque soir à 20 heures des applaudissements dédiés aux personnels soiǵnants. Des musiciennes, des musiciens partaǵeaient des moments de convivialité musicale fenêtres grandes ouvertes, la ville de Montreuil (93) orǵanisa même un «festival des balcons». En Italie, des rues entières chantaient le «Va pensiero» du Nabucco de Verdi ; à Saint-Sébastien, la chanteuse basque Izaro chantait sur le toit du théâtre Victoria Eugenia pour le public des fenêtres ${ }^{53}$. Cette sociabilité musicale faite de précarité, d'improvisation bienveillante cherchait à produire de l'inédit dans l'espace de sidération dans lequel notre monde se trouvait plongé.

Le 3 mai 2020, Weimar, Allemaǵne, les balbutiements d'une reconquête des espaces sonores. Nous habitons le désert des Tartares. Le virus reste une menace. Les musiciens du Bach Collegiium de Thuringe jouent «Bach avec masques» devant un public lui-même soumis aux règles de la distanciation sociale à l'occasion du rassemblement Live Is Life dans le pare du palais du Belvédère. Cet événement qui nous entraîne déjà dans «le monde d'après » fut organisé afin de fournir un soutien financier aux artistes indépendants. Une manière, pour les musiciennes et les musiciens, d'activer, une fois encore, «cette vieille idée humaniste, toujours démentie par l'expérience, jamais récusée pourtant, qui consiste à croire qu'un assaut de beautés et de grandeurs saura braver la méchanceté du monde ${ }^{54}$ ».
41. https://www.silkroad.org/ (consulté le 25 juin 2020)

42. Gérard Lenclud, «Vues de l'esprit, art de l'autre», Terrain 14, 1990: 11.

43. Voir llan Greenberg, «Murder Music», Guernica (magazine new-yorkais dédié à I'articulation entre art et politique) 15 décembre 2010 [en ligne], disponible sur: https://www. guernicamag.com/greenberg_12 15_10/(consulté le 17 juin 2020).

44. Guido Fackler, «Des Lagers Stimme», Musik im KZ: Alltag und Häftlingskultur in den Konzentrationslagern 1933 bis 1936, Brême, Temmen, 2000; Juliane Brauer, Musik im Konzentrationslager Sachsenhausen, Berlin Metropol, 2009.

45. Suzanne Cusick, «Musicology, Torture, Repair»), Radical Musicology 3, 2008; «Toward an Acoustemology of Detention in the Global "War on Terror"», in Georgina Born (dir.),

Music, Sound and Space:

Mransformations of Public and

Private Experience, Cambridge /New York, Cambridge University Press, 2013: 275-291; Katia

Chornik, «Music and Torture in Chilean Detention Centres: Conversations with an Ex-Agent of Pinochet's Secret Police», in Birgit Abels, Barbara Alge, M J Grant et Anna Papaeti (dir.) Music and Torture, Music and Punishment 2 (1), 2013: 51-65.

46. Laurent Feneyrou, De lave et de fer: essai sur l'art des années de plomb en Allemagne Paris, Éditions Mf, 2017.

47. Steve Goodman, Sonic Warfare: Sound, Affect, and the Ecology of Fear, Cambirdge, Massachusetts, MIT Press, 2010.

48. J. Martin Daughtry, Listening to War: Sound, Music and Survival in Wartime Iraq New York, Oxford University Press, 2015

49. Juliette Volcler, Le Son comme arme: les usages policiers et miliaires du son. Paris, La Découverte, 2011.

50. Luis Velasco-Pufleau, «Après les attaques terroristes de l'Éłat islamique à Paris. Enquête sur les rapports entre musique, propagande et violence armée», Transposition 5 2015 [en ligne], disponible sur: 2015 [en ligne], disponible sur: org/transposition/ 1327 (consulté le 09 mai 2020).

51. Luiz Velasco-Pufleau (dir.), Transposition (Son, musique et violence), hors-série 2, 2020 [en ligne], disponible sur: https:// doi.org/10.4000/transposition 3213 (consulté le 17 juin 2020)
52. Paul Veyne, op. cit.

53. https://www.youtube.com/ watch?time_continue $=1723$ \& $\mathrm{v}=\mathrm{or} 8 \mathrm{~V}$ _kimoCE\&feature $=\mathrm{emb}$ _logo (consulté le 25 juin 2020)

54. Patrick Boucheron, Ce que peutl'histoire: leçon inaugurale prononcée le jeudi 17 décembre 2015, Paris, Collège de France, 2016 [en ligne], disponible sur: $\mathrm{http}$ ///books.openedition.org/ cdf/4507

(consulté le 17 juin 2020). 